\title{
Linkage of atmospheric blocks and synoptic-scale Rossby waves: a climatological analysis
}

\author{
By ADRIAN M. ALTENHOFF, OLIVIA MARTIUS*, MISCHA CROCI-M ASPOLI, CORNELIA \\ SCHW IERZ and HUW C. DAVIES, Institute for Atmospheric and Climate Science, ETH Zurich, \\ 8092 Zurich, Switzerland
}

(Manuscript received 15 June 2007; in final form 30 June 2008)

\begin{abstract}
The link between atmospheric blocking and propagating and breaking synoptic-scale Rossby waves (termed $P V$ streamers) are explored for the climatological period 1958-2002, using the ERA-40 re-analysis data. To this end, potential vorticity $(P V)$ based climatologies of blocking and breaking waves are used, and features of the propagating waves is extracted from Hovmöller diagrams. The analyses cover the Northern Hemisphere during winter, and they are carried out for the Atlantic and Pacific basins separately.

The results show statistically significant wave precursor signals, up to $5 \mathrm{~d}$ prior to the blocking onset. In the Atlantic, the precursor signal takes the form of a coherent wave train, emanating approximately $110^{\circ}$ upstream of the blocking location. In the Pacific, a single long-lived (10 d) northerly velocity signal preludes the blocking onset. A spatial analysis is conducted of the location, frequency and form of breaking synoptic-scale Rossby waves, prior to the onset, during the lifetime and after the blocking decay. It reveals that cyclonic streamers are present to the southwest and anticyclonic streamers to the south and southeast, approximately 43\% (36\%) of the time in the Atlantic (Pacific) basin, and this is significantly above a climatological distribution.
\end{abstract}

\section{Introduction}

Atmospheric blocks and synoptic-scale Rossby waves are two of the most striking features of tropopause-level atmospheric flow, and both possess distinctive characteristics. An individual block severely disrupts the background circumpolar flow over a substantial depth of the troposphere, is quasi-steady with a lifetime in excess of $\sim 5 \mathrm{~d}$ and is quasi-stationary, exhibiting comparatively little movement compared with the background flow at upper-level. In comparison the tropopause signature of a synoptic-scale Rossby wave, evident as major lateral undulations of the jet stream, also often impacts upon or is intimately linked to flow development in the lower troposphere, but in contrast to a block, it is usually highly transient and very mobile in nature.

The objective of the current paper is to explore the nature of the relationship between these two distinctive phenomena, and the study's motivation builds upon two specific aspects that arise from examining the phenomena from a potential vorticity $(P V)$ perspective. These two aspects are illustrated in Fig. 1, which shows the instantaneous $P V$ distribution on the $315 \mathrm{~K}$ isentropic surface.

\footnotetext{
${ }^{*}$ Corresponding author.

e-mail: olivia@env.ethz.ch

DOI: $10.1111 /$ j.1600-0870.2008.00354.x
}

The first aspect is the interpretation of a block as an uppertropospheric negative $P V$ anomaly (Schwierz et al., 2004a), which is outlined as the bold black contour in Fig. 1(a). Its amplitude is large enough so that the flow and thermal signatures attributable to the anomaly can extend down to the lower troposphere, and the easterly flow component on its equatorward edge can substantially counter or overcome that of the prevailing westerlies. A comprehensive climatology of such anomalies for the Northern Hemisphere has been compiled (Croci-Maspoli et al., 2007) using ERA-40 data. The results document the spatial distribution of block frequency, thereby complementing previous blocking studies (e.g. Dole and Gordon, 1983; Tibaldi and Molteni, 1990), and, in addition, provide a catalogue of block genesis, movement and lysis in the two major block sectors of the Atlantic and Pacific basins. In essence, from the $P V$ perspective, a block's existence is attributable to the presence of a coherent large-amplitude synoptic-scale anomaly of low $P V$ in the extratropics, and a block genesis requires the generation of the associated anomaly. Again from the $P V$ perspective, the generation must equate to either isentropic advection of low- $P V$ air from low latitudes (cf. Nakamura et al., 1997; De Pondeca et al., 1998; Michelangeli and Vautard, 1998) and/or cross-isentrope flow of air from lower elevation, associated with diabatic processes (Crum and Stevens, 1988; Schwierz, 2001). Thus, the $P V$ framework focuses attention on the necessity of 

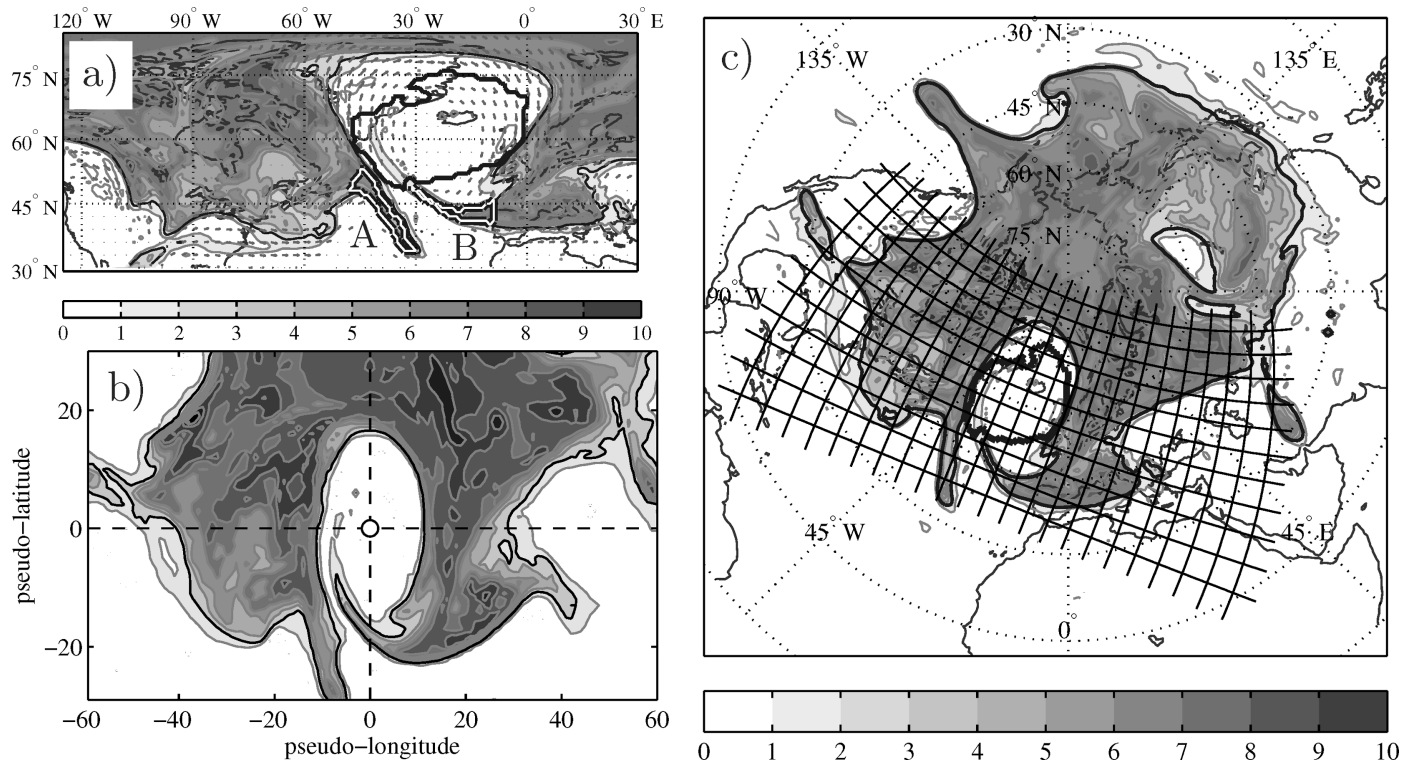

Fig. 1. Illustrative example of a North Atlantic blocking event on 2007 Jan 24, 00 UTC. (a) The grey shading indicates the $P V$ distribution at the $315 \mathrm{~K}$ isosurface (pvu) with the $2 \mathrm{pvu}$ isoline overlaid bold. The isentropic wind vectors on $315 \mathrm{~K}$ are displayed for velocities $>30 \mathrm{~ms}^{-1}$. The bold black contour within the low-PV area encloses the blocked region, whereas the bold black/white contours mark the cyclonically (A) and anticyclonically (B) deformed PV streamer, respectively. (b) The $P V$ field after the rotation of the grid origin to the centre of the block. (c) Northern hemispherical projection of the $P V$ distribution and the block on $315 \mathrm{~K}$ with the rectangular grid overlaid. For better readability PV values have been set to $0^{\circ}$ south of $22^{\circ} \mathrm{N}$. The grid shows the area of the projected situation to the centre of the block.

dynamically and/or thermodynamically redistributing the ambient $P V$ distribution to create a viable blocking pattern, and as such, it differs conceptually from studies (e.g. Shutts, 1983; Vautard and Legras, 1988; Maeda and Tsuyuki, 2000) geared to demonstrate that isentropic advection, linked to high frequency eddies, subject to deformation, can help reinforce a block. A further subtle issue related to the nature of blocks is that empirical evidence (e.g. Mullen, 1986; Nakamura et al., 1997) indicates that blocks in the Atlantic and Pacific exhibit significantly different dynamic characteristics.

The second of the forementioned key aspects relates to the identification of the tropopause-level signature of synoptic-scale Rossby waves, as lateral perturbations of the enhanced $P V$ gradient on tropopause-intersecting isentropes. There are three notable implications of this identification. First is that the wave's propagation can be traced using a refined Hovmöller diagram, which depicts the meridional flow component at the dynamic tropopause (sic. 2 pvu isoline) on a suitable isentropic surface (Martius et al., 2006). The second is that an overturning Rossby wave equates to the dynamic tropopause acquiring an ' $\mathrm{S}$ ' or an 'inverse-S' shaped configuration on an isentropic surface, and the sustained existence of such a configuration has been taken to represent block occurrence (Pelly and Hoskins, 2003). Note that in Fig. 1(a), the block's negative $P V$ anomaly is bounded on its westward and eastward fringes by distortions of the 2 pvu isoline that are akin, respectively, to ' $S$ ' and 'inverse- $S$ ' shaped configurations. The co-existence of these configurations gives the block an overall $\Omega$-shape. The third implication relates to the evolution of the wave(s) beyond the 'S' or 'inverse-S' configuration. Observational case studies (Appenzeller and Davies, 1992), numerical model simulations (Davies et al., 1991; Thorncroft et al., 1993) and climatological compilations (Martius et al., 2007) indicate that the evolution often results in the dilation of the troughs to form narrow elongated streamers of high $P V$.

These typically take one of two paradigmatic arc-shaped forms (Davies et al., 1991; Thorncroft et al., 1993) extending either from the NE to the SW (the anticyclonically curved or LC1 type) or from the NW to the SE (the cyclonically curved or LC2 type). Two $P V$ streamers are highlighted as bold white lines in Fig. 1(a) with the up- and down-stream streamers labelled $\mathrm{A}$ and $\mathrm{B}$, respectively. The counterpart of these equatorward extending stratospheric streamers of high $P V$ (referred to hereafter as STST) are poleward extending tropospheric streamers of low $P V$ (referred to hereafter as TRST). Trenchant points related to the former STSTs are that (1) they can, and often do, undergo a quasi-barotropic instability that, in effect, heralds the final irreversible break-up of the parent Rossby wave (e.g. Appenzeller et al., 1996), and (2) the resulting cut-off $P V$ features are comparatively quasi-steady and quasi-stationary (e.g. Wernli and Sprenger, 2007).

Foundational to the approach adopted herein are the studies of Croci-Maspoli et al. (2007) and Martius et al. (2007). The study of Croci-Maspoli et al. (2007) sets out a method to identify $P V$ 
blocks and records the corresponding climatological distribution for the 1958-2002 period. In Martius et al. (2007), a method is set out to characterise the shape of $P V$ streamers, in line with the LC1 and LC2 classification along with the derivation of the corresponding climatologies.

The foregoing considerations and studies prompt, and make possible the examination of, a sequence of questions: what are the block-resembling combination of $P V$ structures that can counter the ambient extratropical westerly flow and concomitantly enable the block to remain quasi-stationary? What are the dominant observed $P V$ structures that accompany a block at tropopause-levels? Is there, or what is, the characteristic Rossby wave signal that accompanies a block's evolution? Is there evidence that blocks are associated with incipient wave-breaking (sic. appearance of $P V$ streamers) of the LC1 and/or the LC2 type?

In essence, these questions address the validity of the twin hypotheses that (1) block inception is associated with the overturning of a synoptic-scale Rossby wave on isentropic surfaces and (2) the structure resulting from the wave's break-up is integral to maintaining a block's quasi-steady and quasi-stationary character.

These questions and hypotheses form the background to the present study. The paper is organized as follows. First the dynamic issue of a block's quasi-stationarity is considered from a rudimentary $P V$ perspective (Section 2). The blocking and streamer data sets for the study, the compositing approach adopted, and the accompanying significance tests, are set out in Section 3. Thereafter, spatial composites are presented of a block's structure, together with temporal composites of the Rossby-wave signal discernible in Hovmöller diagram during block evolution (Section 5). Likewise composites are provided of the evolving presence of LC1 and LC2 streamers during the block life cycle (Section 6). Insight on the interbasin differences between the Atlantic and Pacific are examined by compiling separate climatologies for each sector.

\section{Rudiments of block dynamics}

The combination of the generation, maintenance and lack of movement of a block poses a dynamic conundrum since it requires a major reconfiguration of the background atmospheric state, and furthermore, the resulting state has to correspond to a spatial structure that has both temporal resilience and is able to counter the effect of the ambient westerly flow. Insight on the requisites for stationarity and the temporal evolution of blocks can be gained with some rudimentary and qualitative inferences drawn from the $P V$ perspective.

\subsection{Stationarity}

The background distribution of $P V$ at tropopause levels equates to a circumpolar flow in the extratropics, with the jet-stream co-
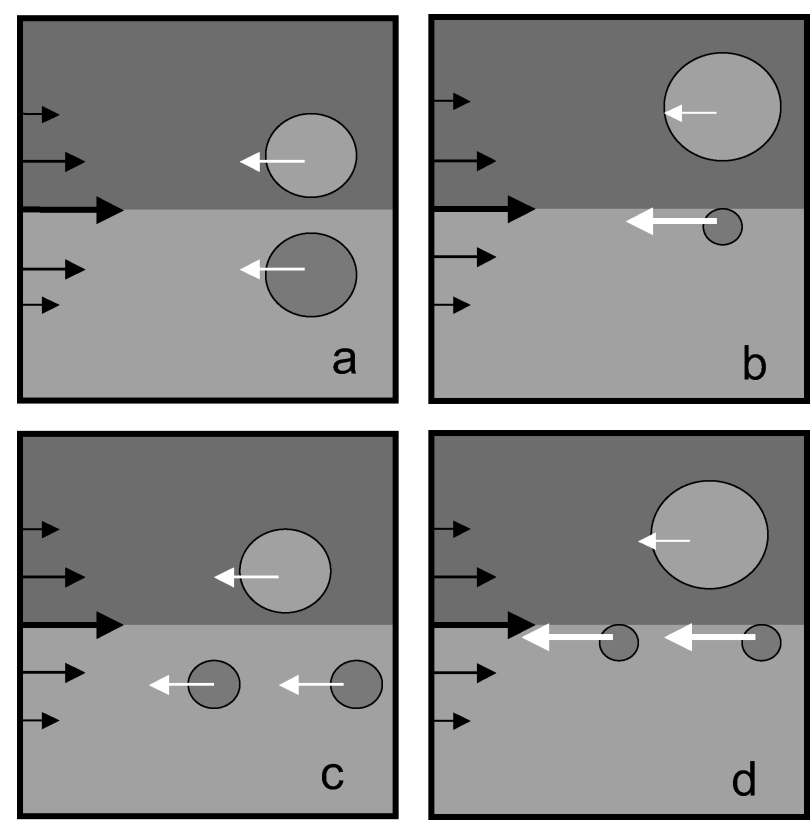

Fig. 2. Schematic isentropic depiction of four highly idealized $P V$ configurations possibly linked to blocked flow. In each panel, the background $P V$ distribution corresponds to a pattern of uniformly high (low) $P V$ located polewards (equatorwards) of a zonally aligned interface, and the circular anomalies correspond to a redistribution of that background $P V$. The upper (a and b) and lower panels (c and d) correspond, respectively, to dipolar and tri-polar PV anomaly distributions, and in the left-hand (right-hand) panels the anomalies are located symmetrically (asymmetrically) relative to the zonal interface. Note also that the black and white arrows indicate, respectively, the jet flow accompanying the background $P V$ distribution and the perturbed flow attributable to the redistributed $P V$.

located with the marked slope of the tropopause height. Thus, a local block-like negative $P V$ anomaly in the vicinity of the jet would be deformed and advected eastwards by the background flow. To retain its original longitude, it is necessary to counter this background flow with a richer $P V$ distribution.

Simple schematic examples of possible $P V$ distributions on an isentropic surface that match this requirement are shown in Fig. 2. In each case the background $P V$ distribution is assumed to be a two-zone distribution, comprising high $P V$ to the north of the jet and low $P V$ to the south (cf. Swanson et al., 1997; Schwierz et al., 2004b).

In the upper panels (Figs. 2a and b), there are dipolar patterns of $P V$ anomalies, located symmetrically and asymmetrically across the jet. In the former case (Fig. 2a), the anomalies are of equal strength/area, and the influence of each anomaly upon another counters the ambient flow, whereas, together they generate a stronger easterly flow at the latitude of the jet. In the asymmetric setting (Fig. 2b) the strength/area of the two anomalies differ so as to counter the latitudinally varying strength of the background westerly flow. In the lower panels (Figs. 2c and d), there are tri-polar $P V$ distributions, centred at the corners 
of an equilateral triangle, with the negative poleward anomaly assuming twice the strength/area of the other two. Again, the influence of the neighbouring vortices upon one another offsets the ambient flow. These simple heuristic considerations lead to instructive inferences. They suggest the corroboration of the following hypothesis:

(1) A block's negative $P V$ anomaly needs to be accompanied by a neighbouring positive anomaly or anomalies (the alternative of a localized and stationary synoptic-scale wave packet appears less plausible).

(2) There are amplitude and geometric constraints upon the $P V$ anomalies related to the strength and sharpness of the background jet.

(3) The two simplest conceivable patterns bear comparison with so-called cut-off and $\Omega$-shaped blocks ( $c f$. the differing background settings and preferred block configurations in the North Atlantic and Pacific).

\subsection{Evolution}

Three inferences follow, if the idealized distributions in Fig. 2 are deemed to be indicative of realized blocks. First, a major distortion of the jet would, in the absence of diabatic effects, constitute the most likely origin of the requisite anomalies. Second, an overturning Rossby wave would equate to an incipient dipole distribution, but further conditions would need to be met to produce relative stationarity of the block and/or limit the occurrence of excessively strong easterlies at the centre of the bipolar distribution. Third, the overturning of a Rossby wave resembles an incipient dipolar structure, and the co-occurrence of an LC1 and LC2 structure would connote a tripolar $\Omega$-pattern.

In a realized flow evolution the negative $P V$ anomaly of a block and an evolving $P V$ streamer can interact non-linearly and synergetically. For example, the $P V$ streamer and the accompanying trough, labelled A in Fig. 1(a), can increase a block's $P V$ anomaly in two ways. First, it can help advect subtropical low$P V$ air quasi-isentropically towards and, in some cases, into the blocking area along the $P V$ streamer's eastern flank. This adds to the low- $P V$ advection that is observed in an earlier linear stage of the life cycle of baroclinic waves (Nakamura and Wallace, 1993). Second, the $P V$ streamer is also often associated with surface development, and the associated cloud-diabatic transfer of air polewards and upwards from the lower troposphere can deposit low- $P V$ air in the downstream ridge. Likewise, the block can, in turn, contribute to the generation of a streamer down-stream on its eastern flank (e.g. Massacand et al., 2001).

Contrariwise an incipient block and a pre-existing downstream streamer ( $c f$. streamer B in Fig.1a) can together help deform a trough incident from upstream to form a meridionallyelongated upstream $P V$ streamer. In both cases, the result would be a pronounced $\Omega$-shaped block akin to that evident in Fig. 1(a).
In the remainder of the study, the occurrence of the sequence of events alluded to above is examined by exploiting the availabile $P V$-blocking, $P V$-streamer and Rossby wave climatologies.

\section{Data}

This study uses the ERA-40 re-analysis data set (Uppala et al., 2005) from the European Centre for Medium-Range Weather Forecast (ECMWF). The data set covers the time period from December 1957 to February 2002. The analysis have been carried out for both the satellite era (1978 to 2002) and the entire ERA-40 time period. The results do not differ significantly, and therefore, only the results for the entire time period are discussed hereafter. The fields are available 6 hourly and the data are interpolated horizontally onto a $1^{\circ} \times 1^{\circ}$ Gaussian grid from the T159L60 spectral model output.

The three climatologies described hereafter have all been computed using the three-dimensional hydrostatic form of the Ertel $P V$ field.

(1) The two-dimensional atmospheric blocking climatology (Croci-Maspoli et al., 2007) is derived using a $P V$-based blocking indicator (Schwierz et al., 2004a). This indicator captures coherent and sustained negative $P V$ anomalies at tropopause elevations that constitute the core $P V$ contribution to a block. These negative $P V$ anomalies are tracked, and only anomalies with lifetimes longer than $5 \mathrm{~d}$ are identified as blocks. The final blocking climatology data set consists of 6-hourly geographical representations of blocked or non-blocked areas. Note that, herein, the terms blocking onset/decay represent the first/last time a block is detected with this index.

(2) Synoptic-scale Rossby waves are identified from a climatology of $P V$-waveguide Hovmöller diagrams. These Hovmöller diagrams differ from regular ones in that they show the meridional wind velocity on middle world isentropic surfaces (310 to $350 \mathrm{~K}, 5 \mathrm{~K}$ intervals) averaged along the dynamic tropopause ( 2 pvu isoline) rather than within a latitudinal belt (Martius et al., 2006).

(3) The $P V$ streamer climatology (Martius et al., 2008) on middle world isentropic surfaces $(310$ to $350 \mathrm{~K}, 5 \mathrm{~K}$ intervals) has been calculated, using the methodology of Wernli and Sprenger (2007). $P V$ streamers are identified along the dynamic tropopause as elongated structures with a narrow neck on an isentropic surface. Two types of $P V$ streamers are identified: STST, corresponding to stratospheric intrusions extending equatorward and downward into the troposphere, and TRST, corresponding to poleward and upward tropospheric intrusions into the stratosphere. The resulting $P V$ streamer data set consists of 6-hourly gridded binary fields that indicate the location of the streamers. As mentioned earlier, the streamers are grouped into two classes using the orientation of the main streamer axis (Martius et al., 2007) such that NW-SE extending streamers are referred 

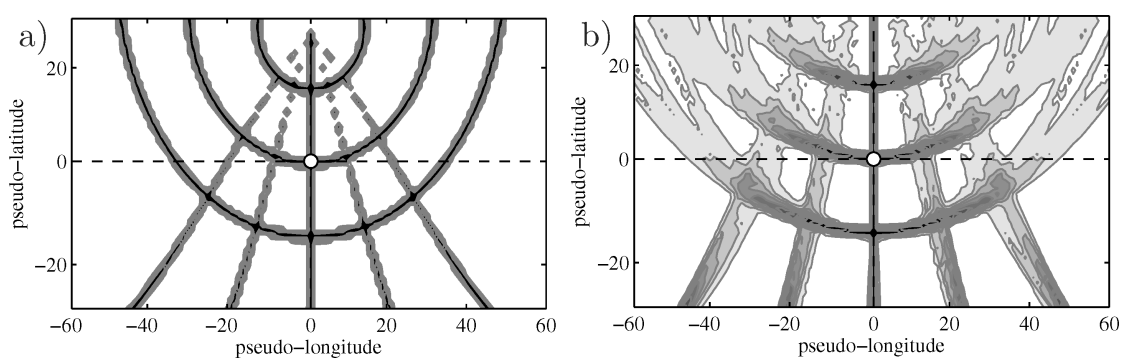

Fig. 3. (a) Geographical grid for the case example illustrated in Fig. 1 after the rotation; (b) frequency distribution of the rotated geographical grids (longitude, $10^{\circ}$ steps; latitude, $15^{\circ}$ steps) for the onset time of all Atlantic blocking cases. The shading indicates the probability that the original grid lines (longitude, $10^{\circ} \mathrm{steps}$; latitude, $15^{\circ}$ steps) are located at a specific point in the new coordinate system after the rotation $(0-0.5, \Delta 0.05$ interval).

to as cyclonic or LC2-type streamers and NE-SW orientated streamers are called anticyclonic or LC1-type streamers.

\section{Methodology}

The co-occurrence of atmospheric blocking, synoptic-scale Rossby waves and $P V$ streamers is analysed separately for the North Atlantic $\left(90^{\circ} \mathrm{W}-50^{\circ} \mathrm{E}, 30^{\circ} \mathrm{N}-80^{\circ} \mathrm{N}\right)$ and North Pacific $\left(110^{\circ} \mathrm{E}-110^{\circ} \mathrm{W}, 30^{\circ} \mathrm{N}-80^{\circ} \mathrm{N}\right)$ regions. For the given time period, the number of identified winter (DJF) blocking events in these regions are, respectively, 212 for the North Atlantic and 198 for the North Pacific.

\subsection{PV composites}

First, regional $P V$ composite maps are derived for the blocking events. Recognizing the high spatial case-to-case variability of block location, a refined composite analysis is introduced that displays the $P V$ field in a framework relative to the block centre. In effect the 6 hourly fields are projected onto a rectangular grid whose origin coincides with the centre of mass of the block. This is done using an algorithm, commonly applied to transform grids for limited area model simulations. The effects of the procedure on a sample blocking event are illustrated in Figs. 1 and 3. Figure 1(b) shows the transformed $P V$ pattern, corresponding to that shown in the conventional cylindrical projection in Fig.1(a), and Fig. 1(c) shows the location of the new rectangular grid within the geographical grid. Likewise Fig. 3(a) shows the transformed geographical grid for the sample case. Note that the curvature of the original geographical grid in the new projection is dependent on the latitudinal position of the block. Hence, the variations in the latitudinal position of the individual blocks translate into distortions that are evident in the far field of the rectangular grid. A composite plot of the transformed geographical grids for all North Atlantic blocking events serves to visualize the smearing in the far field (Fig. 3b).

In consequence, only a domain of approximately $40^{\circ}$ around the block is used in this study. In essence, the method portrays the $P V$ pattern relative to the block. Nevertheless, tentative general statements about the geographical background flow can be made because the frequency maxima of blocks are located in welldefined and spatially restricted areas on a basin-size scale (CrociMaspoli et al., 2007, Fig. 2).

\subsection{Synoptic-scale Rossby waves relative to the blocks}

The approach of Martius et al. (2006) is adopted and adapted to derive the composite signal of Rossby waves centred on the onset and decay of the block. The two composite Hovmöller time-traces are prepared by shifting the signal to the longitude of the blocking centre at the time of, respectively, the onset and decay, and this is done prior to compositing.

To test the significance a Monte Carlo method is used. In this procedure 500 random Hovmöller-composites are calculated and used to identify deviations of the blocking Hovmöllercomposites that are significant on the two-sided $99 \%$ percentile level when compared with the random sample.

\subsection{PV streamer relative to the blocks}

In a final step, regional composite maps are produced of the $P V$ streamer occurrence and location relative to the block. Again the fields are transformed onto the same local plane projection. For this analysis, the composite feature is the vertically-integrated streamer frequencies. The reason for adopting this parameter are twofold: (1) the block itself is a three-dimensional, and quasibarotorpic feature over the isentropic levels considered here (e.g. Schwierz et al., 2004a) and a vertical integration takes this aspect into account, and (2) it is not a priori clear on which isentropic level the link between the block and the streamers can be expected to be the strongest.

The integrated frequencies are calculated as follows. In a first step, all gridpoints are flagged as a streamer point, where a streamer is detected on one of the levels between 310 and $350 \mathrm{~K}$. In a second step, these fields are temporally averaged over $2 \mathrm{~d}$.

These composites (using 6 hourly data) are calculated starting (1) $2 \mathrm{~d}$ before and ending $6 \mathrm{~d}$ after the blocking onset and (2) starting $6 \mathrm{~d}$ before the blocking decay and ending $2 \mathrm{~d}$ after block disappearance.These composites will be referred to as spatial $P V$ streamer frequency composites and, again, are tested for 

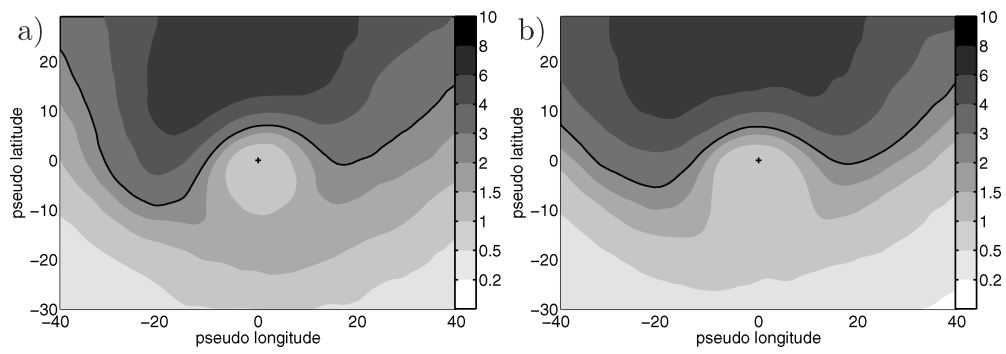

Fig. 4. Composite maps of isentropic $P V$ on $310 \mathrm{~K}$ averaged over the first $2 \mathrm{~d}$ after the blocking onset in (a) the North Atlantic and (b) the North Pacific region, respectively. The fields are rotated such that the mean centre of the block is the centre of the coordinate system (black cross). The solid black line indicates the 2 pvu isoline. significant deviations from the climatological streamer distribution. To this end, a standard re-sampling Monte Carlo test is applied. First 500 random $P V$ streamer composites are calculated for the number of regionally blocked days. Then the two-sided $95 \%$ percentile level of this 500 member sample is used as confidence interval.

\section{5. $P V$ and Rossby wave composites}

\subsection{PV composites}

$P V$ composites, incorporating all block episodes detected in the data base, were compiled separately for the two ocean basins. The composites were prepared for isentropic levels from $3100 \mathrm{~K}$ to $350 \mathrm{~K}$. Figure 4 shows the results on $310 \mathrm{~K}$ and indicates a distinct interbasin difference, even for the composite structures.

In the North Atlantic composite, the pattern has a cut-off low- $P V$ anomaly, located well to the north of the upstream zone of enhanced $P V$ gradient (Fig. 4a). The pronounced upstream and downstream troughs are indicative of strong wave activity, but the composite approach smears out the signal of streamers occurring in disparate locations (see Section 5 for the streamer climatology). In the Pacific (Fig. 4b), the block composite is characterized by a northward intrusion of low- $P V$ air from the subtropics and an overall smoothed $\Omega$-shape structure.

In summary, the $P V$ composites, more specifically the resulting anomalies when subtracting the near zonally-symmetric background $P V$ field, bear comparison with the schematic displays in Fig. 2. The patterns in the North Atlantic and Pacific appear to resemble, respectively, the asymmetric and symmet- ric configurations. Likewise, the cut-off North Atlantic pattern equates more closely the dipolar structure, whereas the North Pacific pattern is more akin to a tripolar equilateral distribution of $P V$ anomalies.

\subsection{Rossby wave composites}

Composite Hovmöller diagrams of the meridional wind component along the dynamic tropopause can provide a first indication of the characteristic space-time evolution of the Rossby wave signal, accompanying blocking episodes. The compositing will highlight waves of various spatial scales that are in-phase with each other, and conversely, lead to a cancellation of waves that are not in phase. Separate composites are prepared that are centred in time on the block onset and block decay, respectively, and displayed for both the North Atlantic and Pacific (Fig. 5). Only the significant (99\% percentile) portions of the composite are displayed in the subsequent time traces.

5.2.1. Block onset. In the North Atlantic (Fig. 5a), a positive peak in the meridional velocity is present on $320 \mathrm{~K}$ surface at approximately $110^{\circ}$ upstream of the block formation longitude, $5 \mathrm{~d}$ prior to the blocking onset. The signature is deep in vertical extent (evident on isentropic surfaces between 310 and $340 \mathrm{~K}$, not shown) and persists for approximately $5 \mathrm{~d}$. Initially, it retains an almost constant longitudinal location, but begins to translate eastwards at day -3 . Between day -3 and day -2 , a weak negative peak appears at $70^{\circ}$ upstream of the block.

Thereafter, a positive peak appears, which is initially located upstream of the block onset longitude and a negative peak appears downstream of the onset longitude. These two peaks are,
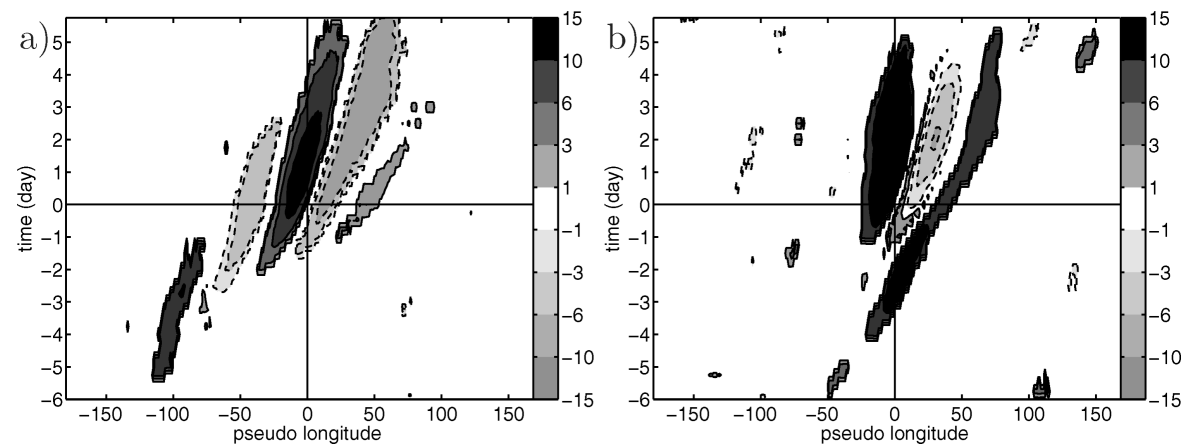

Fig. 5. Hovmöllerdiagram of composites of the meridional wind on $320 \mathrm{~K}$ along the dynamic tropopause from $6 \mathrm{~d}$ prior to $6 \mathrm{~d}$ after blocking onset for (a) the North Atlantic and (b) the North Pacific region. 

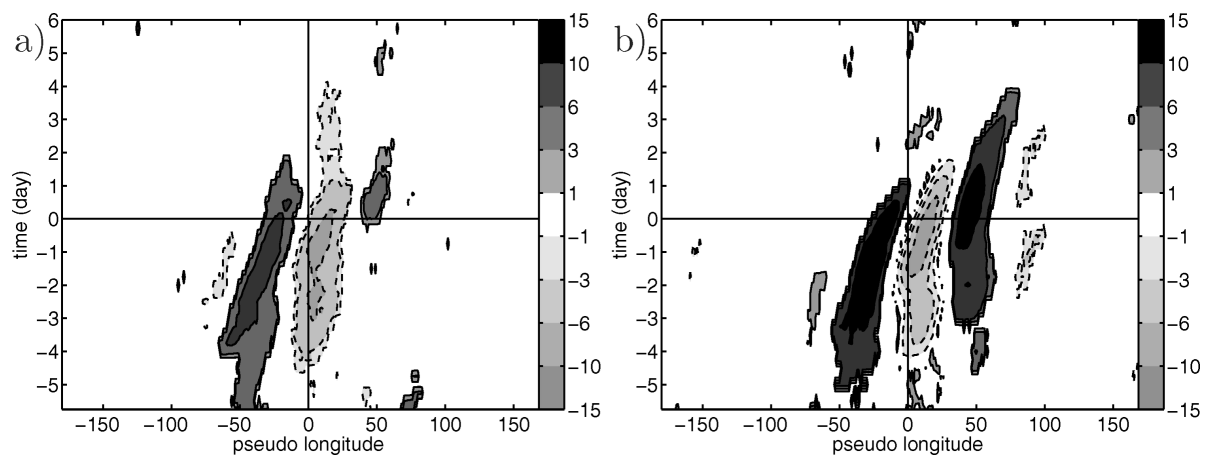

Fig. 6. As for Fig. 5 but during the blocking decay phase.

in effect, the block's meridional velocity signal. The eastward translation of these two peaks decreases at block onset, and this decrease is stronger for the upstream component, indicating a zonal widening of the block thereafter. A weak positive peak also appears downstream of the block, around the onset, translates significantly faster than the block and is evident for $2 \mathrm{~d}$.

In the North Pacific, on the other hand, there is no evidence of a coherent in-phase upstream precursor signal on $320 \mathrm{~K}$ composite (Fig. 5b). A positive meridional velocity signal is evident on day -4 at the longitude of the subsequent block. It persists for almost $10 \mathrm{~d}$ and translates eastwards at approximately $15^{\circ}$ per day up until day +2 . This takes it well to the east of the block longitude, and thereafter, it becomes almost stationary up to day +5 . This downstream signal has a deep vertical and meridional extent (evident on theta-surfaces between 310 and $330 \mathrm{~K}$, not shown). In the meanwhile, the block signal of juxtaposed positive and negative bands appears at day -1 , persist to beyond day 5 , and the relative eastward translation of the bands is indicative of a widening of the block.

5.2.2. Block decay. During the decay phase the signatures in the neighbourhood of the block again differ markedly between the Atlantic and Pacific (Fig. 6), and this is particularly true downstream of the block's decay longitude.

In the Atlantic basin (Fig. 6a) the block signature of positive (upstream) and negative (downstream) peaks effectively disappear at the block decay time, with a weak positive peak appearing transiently (order of $2 \mathrm{~d}$ ) downstream.

In the Pacific basin (Fig. 6b), there are three significant signals. There are the anticipated quasi-stationary positive and negative signals of the blocks that are initially quasi-stationary but begin to translate eastwards and weaken rapidly around the decay time (Note that the positive component is much stronger than its Atlantic counterpart). In addition, a positive wave peak emerges downstream of the block $3 \mathrm{~d}$ prior to the decay time, lasts for approximately $7 \mathrm{~d}$ and translates significantly eastwards after the decay time.

5.2.3. Discussion. The precursor signal ahead of the onset time in the Atlantic conforms to a classical downstream wave development (e.g. Chang et al., 2002). It is noteworthy that the wave peak's longitudinal phase velocity decreases substantially $1 \mathrm{~d}$ ahead of and after onset. The waves become quasi-stationary as observed in Nakamura et al. (1997) and Nakamura and Fukamachi (2004). The wavelength of the trough upstream of the blocking ridge decreases substantially, immediately ahead of and after onset. This is consistent with a scale-contraction of the incoming synoptic-scale wave, associated with the so-called eddy-straining mechanism (Hoskins et al., 1983; Shutts, 1983). At the onset time, the composite is consistent with a troughridge-trough structure dominated by the ridge, but by day +3 , the signal is reduced essentially to a single ridge. In contrast, the decay in the Atlantic is not, at least in the composite framework, accompanied by a downstream wave transmission.

Almost the opposite prevails in the Pacific. In the onset phase, there is no evidence of a coherent upstream precursor signal, rather in situ a contemporaneous appearance of the block signature at around onset. This can be interpreted in two ways. The synoptic-scale waves upstream of the Pacific blocks could be out of phase, and hence, their signal is smeared out during the composition, or the waves are either of very small amplitude or completely absent. It has been argued (e.g. Nakamura, 1992) that the strong jet stream, upstream of the preferred blocking region over the Pacific, is not conducive to large-amplitude upstream development. In the decay phase, there is evidence of a longitudinal/downstream emittance of a wave energy (cf. Nakamura, 1994; Nakamura et al., 1997).

\section{Streamer composites}

Here we examine the $P V$ streamer frequencies in the neighbourhood of blocks, for the time periods centred first on block onset and then on block decay. In particular, attention is directed at significant deviations of the streamer frequencies during blocked days from the corresponding climatological distributions.

\subsection{Blocking onset}

Figure 7 shows the temporal evolution of the spatial STST frequencies during the first $6 \mathrm{~d}$ of blocking. Only areas where the 

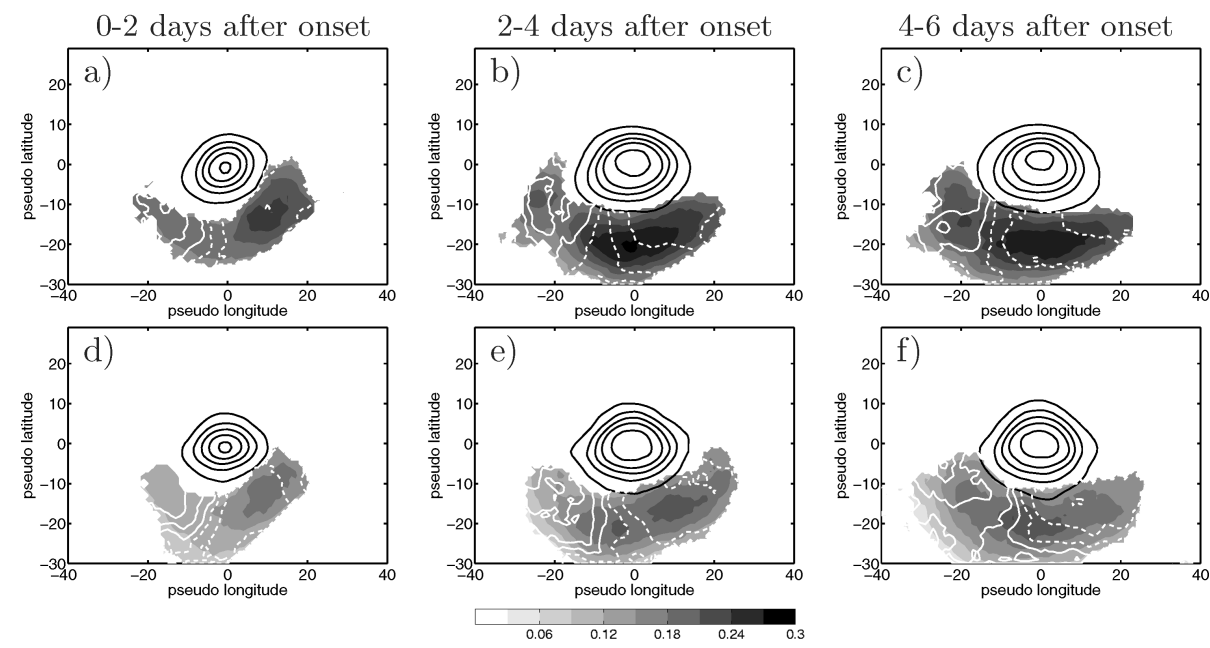

Fig. 7. Composite maps of atmospheric blocking (black lines) and the corresponding spatial distribution of frequency of stratospheric $P V$ streamers (shaded) during the blocking onset and growth phase for (a)-(c) the North Atlantic and (d)-(f) the North Pacific region, respectively. The mean blocking frequency is indicated in black-circled contours ( 0.9 to 0.1 with 0.2 spacing). In addition, the ratio between cyclonically and anticyclonically deformed $P V$ streamers is indicated by the white contours, where the thick line indicates the equal ratio 1 and the contour spacing equals $5: 1,4: 1,2: 1,4: 3,1,3: 4,1: 2,1: 4,1: 5$. Dashed contours indicate a majority of anticyclonic $P V$ streamers.

spatial STST frequency significantly exceeds the climatological distribution (95\% significance level) are displayed, and as noted earlier, the layout of the figure is such that the block is always located in the centre of the plot and the streamer locations adjusted accordingly (cf. Section 4).

For the interpretation of Fig. 7, it is important to note that (1) areas where the spatial STST frequency is significantly below the climatological distribution are all located within the blocking area (not shown) and are not discussed further; (ii) prior to the blocking onset (days -2 to 0 ), the spatial STST frequencies in the vicinity of the block do not differ significantly from the climatological distribution (not shown).

Between day 0 and day 2 after the onset of the block, a STST frequency maximum is located to the southeast of the block both in the Atlantic and the Pacific composite (Figs. 7a and d), and the significant area extends to the southwest of the block. The fraction of cyclonic (LC2, indicated by the white contours) streamers is highest to the southwest of the block, where more cyclonic than anticyclonic (LC1) streamers are found. Between day 2 and 4 (Figs. $7 b$ and e), the spatial streamer frequency maximum is shifted to the south of the block in both the Pacific and the Atlantic basin. During this period a more pronounced separation takes place into a majority of anticyclonic streamers in eastern part and more cyclonic streamers in the western part of the significant area. On day 4-6 (Figs. 7c and f), the amplitude and spatial extent of the streamer maxima remain approximately the same as on days 2-4. It is notable that for the entire onset phase, (1) the spatial streamer frequencies are generally lower in the Pacific basin than in the Atlantic by about $15-30 \%$ and (2) the fraction of cyclonic type streamers is higher in the Pacific.

The above results are confirmed by an additional analysis indicating the percentage of blocking cases where an STST is present in the vicinity of the block (within a radius of $4000 \mathrm{~km}$ ). Note is also taken of the streamer's location up- or down-stream of the block. The resulting percentage is normalized by the total number of time steps when a block is occurring.

Averaged over the entire onset phase, STST are detected in the vicinity of blocks approximately $43 \%$ of the time in the Atlantic and $36 \%$ of the time in the Pacific. In the Atlantic, for about $20 \%$ of the time, anticyclonic and cyclonic STST occur together. The most frequent configuration (12\%) consists of a cyclonic STST located upstream and an anticyclonic STST located downstream of the block. Cyclonic (anticyclonic) STST alone are detected in about $14.5 \%$ (9.5\%) of the time, and for this 'single' configuration, anticyclonic streamers are located, again, preferentially downstream of the block and cyclonic streamers preferentially upstream of the block. Further common combinations are two cyclonic streamers located upstream and downstream of the block, respectively.

In effect all possible combinations of anticyclonic and cyclonic streamers located either up- and/or downstream of the block are observed. The findings in the Pacific differ in that cyclonic streamers that occur alone $(17 \%)$ are more frequent than the occurrence of both streamer types together (11\%).

\subsection{Blocking decay}

The composites for the decay phase of the block are shown in Fig. 8. The three panels for the Atlantic (Figs. 8a-c) and Pacific (Figs. 8d-f), respectively, correspond to two-day means, starting on day 6 before the decay. After the decay, no significant streamer signals are found (not shown). The streamer frequency maximum during blocking decay is located to the south of the block as during the onset phase. 

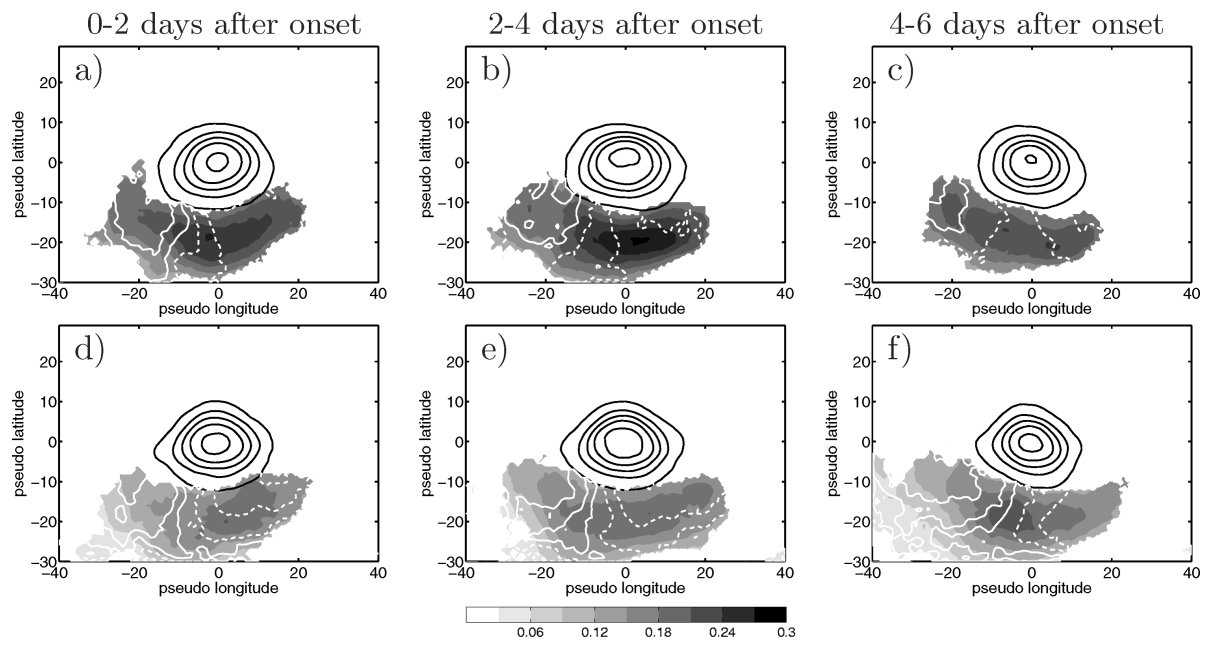

Fig. 8. As for Fig. 7 but during the decay phase of the block.

Again for this phase, streamer frequencies in the Pacific basin are about $30 \%$ less than those in the Atlantic. In the Atlantic, the relative fraction of cyclonic streamers decreases with increasing age of the block, and the frequency of the streamers decreases prior to the decay of the block. In the Pacific on the other hand, the fraction of cyclonic streamers upstream of the block remains constant prior to the decay, and the absolute frequency of the streamers increases slightly.

\subsection{Discussion}

The above analyses provide a quantitative and basindiscriminating description of the co-occurrence of STST and blocks. Here, we comment further on the result that approximately $43 \%$ (Atlantic) and 36\% (Pacific) of blocks are linked to STST, or the obverse that, respectively, $57 \%$ and $64 \%$ of the blocks are not linked to STST during their onset phase.

This result could be attributable to several factors. Preeminently streamer-like features might merely be completely absent in the neighbourhood of the block. For example, classical $\Omega$ shaped blocks could evolve without the occurrence of wavebreaking. The smaller STST frequencies detected in the Pacific, support this interpretation since $\Omega$-type blocks have been observed more frequently in the Pacific than in the Atlantic (e.g. Mullen, 1986; Nakamura et al., 1997). Another reason for the relatively low percentage of blocks and STST co-occurrence lies in the nature of the STST detection routine. The routine detects only overturning waves in the final stage of the baroclinic life cycle, with a thin connection to the main stratospheric air body. Hence, waves that take the form of relatively broad trough-like structures, will not be detected as STST.

The results of the composite analyses support the hypothesis stated in the introduction that $P V$ streamers can contribute to blocking intensification and that blocks, in turn, can influence the downstream wave -overturning. First, the high frequency of anticyclonic STST found downstream of the blocks is consistent with the large-scale deformation associated with the block. Second, the high frequency of cyclonic STST upstream of the block is a hint to the possible combined contributions of advective and diabatic processes to block formation intensification, since this type of wave-breaking is linked to strong uplift from the boundary layers (Polvani and Esler, 2007) and strong (Davies et al., 1991), and sometimes explosive, cyclogenesis (e.g. Colucci, 1985) with concomitant strong diabatic effects.

Note that a similar analysis (not shown) has been undertaken for TRST intrusions, corresponding to low- $P V$ air injection from the subtropics towards the pole. In the Atlantic basin, no significant tropospheric streamer signal can be detected prior to the blocking onset. After the onset, the spatial frequencies of TRST in exceed $30 \%$ in the core of the block, indicating the importance of poleward intrusions for the maintenance of the blocking. In the Pacific basin, the percentage is slightly lower.

Furthermore, by subdividing the tropospheric streamers into anticyclonic and cyclonic classes, it is possible to determine the fraction of anticyclonic TRST at blocking onset. In both the Atlantic and the Pacific basins, the anticyclonic streamers are more frequent; however, the percentage of cyclonic streamers is substantially higher in the Pacific $(3: 4)$ than in the Atlantic $(1: 4)$. This observed TRST orientation lends credence to the hyptheses of Swanson (2001), based upon idealized model simulations that identifies two types of blocking onsets-an anticyclonic and a cyclonic one. He states that the cyclonic onset type is found more often in the Pacific basin whereas the anticyclonic cases occur more frequently in the Atlantic.

It is pertinent to recall that there are methodological restrictions to the composite analyses. First, the spatial domain considered is confined longitudinally, due to distortion introduced by the spatial transformation, performed to derive the composite fields. In effect, this prohibits an analysis of the 
composite up- and downstream development to approximately $\pm 40^{\circ}$ away the blocking centre. Second, the relatively strict constraints imposed upon the form of $P V$ streamer by the stipulated detection routine permits detection only of waves in the very final stage of their life cycle and concomitantly omits relatively broad troughs, which might also influence the block.

\section{Summary and conclusion}

In this study, three novel and independent $P V$-based climatologies of atmospheric blocking, $P V$-streamers and synopticscale Rossby waves are combined to explore the links between synoptic-scale Rossby waves and blocks on the Northern Hemisphere over a 44-year time period.

First, the presence and character of synoptic-scale Rossby waves in the vicinity of a block location are examined prior to the onset, during the lifetime and after the decay of a block. This is done, using Hovmöller diagrams of the meridional wind velocities along the extratropical dynamic tropopause. For the onset phase in the Atlantic, a coherent precursor wave train is found, emanating approximately $110^{\circ}$ upstream of and at approximately $5 \mathrm{~d}$ prior to block onset. In contrast, in the Pacific, an in situ positive wave signal, $3 \mathrm{~d}$ prior to the blocking onset, preludes block occurrence. During the decay phase significant wave signals are detected downstream of the block, at the time of the blocking decay in the Atlantic, and these are more persistent and of larger amplitude in the Pacific.

Second, an analysis is undertaken of the co-occurrence of atmospheric blocking and STST, with the latter viewed as incipiently breaking synoptic-scale Rossby waves. The analysis shows that during the entire blocking lifetime, the spatial frequency of STST is significantly above the climatological distribution to the south of the block. During the block's life cycle, the frequency maxima of the streamers shift from being located southeast of the block (day 0-2 after onset) to being positioned south of the block (day 4-6 after onset), in both the Atlantic and Pacific basins. Generally anticyclonic (LC1) streamers are located more frequently to the southeast of the block and cyclonic (LC2) streamers favoured the southwest. The spatial STST frequencies are about $15-30 \%$ higher in the Atlantic than in the Pacific basin. The most common streamer configuration in the Atlantic is the co-occurrence of a cyclonic STST, upstream, and an anticyclonic STST, downstream of the block, whereas the favoured distribution in the Pacific is a single cyclonic STST, upstream of the block. Streamers of both life-cycle types are found in all possible combinations, being located up- and/or downstream of the block.

Prior to block onset and after block decay, the streamer frequencies are neither significantly above nor below the climatological mean. Nevertheless, the spatial distribution and the orientation of the streamers in the vicinity of the block are consistent with the hypothesis that streamers located upstream of the block enhance the latter by advection of low- $P V$ air from the subtropics and set the scene for cloud-diabatic heating with the possible injection of low- $P V$ air into the upper-troposphere. A block, in return, can exert a crucial influence on a streamer downstream, by distorting and stretching it anticyclonically.

Second, the combined analysis of streamers and waves yields a more integrated picture of the wave-block interaction. The Hovmöller plots offer a detailed analysis of up- and downstream wave development, accompanying blocking. The streamer analysis enables the quantification of: the percentage of blocks associated with breaking waves and the LC1/LC2 classification of the breaking waves. Thus, the two analyses provide complementary results. The different wave signal detected downstream in the Atlantic and Pacific (Fig. 6a) signify that the corresponding differing block-streamer spatial configurations are, respectively, lees and more conducive to generating downstream conditions favouring wave-propagation.

The wave signals prior to the blocking onset and the concomitant absence of significant streamer signals indicate that primarily, comparatively weak synoptic-scale waves play an important role in the onset phase of the blocks.

Significant differences between blocks in the Atlantic and the Pacific basin are found in the mean tropopause level $P V$ field (Fig. 4), the Rossby wave signal (Figs. 5 and 6) and the number of $P V$ streamers (Figs. 7 and 8) prior to the blocking onset and after its decay. From the $P V$ standpoint, these differences are presumably linked to differing ambient planetary-scale flow (e.g. the spatial distribution of stationary troughs and ridges) and deformation fields and/or to a differing potential to inject lowlevel, low- $P V$ air into the upper troposphere via cloud-diabatic processes.

The smaller number of anticyclonic streamers that form downstream of the block in the Pacific, can potentially be explained within the framework of cyclonic versus anticyclonic vortex control, as suggested by Orlanski (2003). In the Pacific, stronger baroclinicity favours a cyclonic vortex control, and hence, the breaking waves would preferentially adopt a cyclonic shape. Whereas in the Atlantic basin, the weaker surface baroclinicity would be associated with a stronger anticyclone and hence an anticyclonic deformation of the streamers in the surroundings of the block.

\section{Acknowledgments}

The authors would like to thank MeteoSwiss for granting access to the ERA-40 data set of the ECMWF. Particular thanks go to Michael Sprenger for providing support with the map transformation routine and to Linda Schlemmer for the inversion data. This research has been supported in part by the Swiss NCCR Climate program.

\section{References}

Appenzeller, C. and Davies, H. C. 1992. Structure of stratospheric intrusions into the troposphere. Nature 358, 570-572. 
Appenzeller, C., Davies, H. and Norton, W. 1996. Fragmentation of stratospheric intrusions. J. Geophys. Res.-Atmos. 101, 1435-1456.

Chang, E. K. M., Lee, S. Y. and Swanson, K. L. 2002. Storm track dynamics. J. Clim. 15, 2163-2183.

Colucci, S. J. 1985. Explosive cyclogenesis and large-scale circulation changes: implications for atmospheric blocking. J. Atmos. Sci. 42, 2701-2717.

Croci-Maspoli, M., Schwierz, C. and Davies, H. 2007. A multi-faceted climatology of atmospheric blocking and its recent linear trend. $J$. Clim. 20, 633-649.

Crum, F. X. and Stevens, D. E. 1988. A case-study of atmospheric blocking using isentropic analysis. Mon. Wea. Rev. 116, 223-241.

Davies, H. C., Schär, C. and Wernli, H. 1991. The palette of fronts and cyclones within a baroclinic wave development. J. Atmos. Sci. 48, 1666-1689.

De Pondeca, M. S. F. V., Barcilon, A. and Zou, X. 1998. The role of wave breaking, linear instability, and PV transports in model block onset. J. Atmos. Sci. 55, 2852-2873.

Dole, R. M. and Gordon, N. D. 1983. Persistent anomalies of the extratropical Northern Hemisphere wintertime circulation - geographicaldistribution and regional persistence characteristics. Mon. Wea. Rev. 111, 1567-1586.

Hoskins, B. J., James, I. N. and White, G. H. 1983. The shape, propagation and mean-flow interaction of large-scale weather systems. $J$. Atmos. Sci. 40, 1595-1612.

Maeda, S. and Tsuyuki, T. 2000. Relationship between singular modes of blocking flow and high-frequency eddies. J. Meteorol. Soc. Jpn. 78, 631-646.

Martius, O., Schwierz, C. and Davies, H. C. 2006. A refined Hovmöller diagram. Tellus 58A, 221-226.

Martius, O., Schwierz, C. and Davies, H. C. 2007. Breaking waves at the tropopause in the wintertime Northern Hemisphere: climatological analyses of the orientation and the theoretical LC1/2 classification. $J$. Atmos. Sci. 64, 2576-2592.

Martius, O., Schwierz, C. and Sprenger, M. 2008. Dynamical tropopause variability and potential vorticity streamers on the northern hemisphere: a climatological analysis. Adv. Atmos. Sci. 25 No 3, 367-369.

Massacand, A., Wernli, H. and Davies, H. 2001. Influence of upstream diabatic heating upon an Alpine event of heavy precipitation. Mon. Wea. Rev. 129, 2822-2828.

Michelangeli, P. A. and Vautard, R. 1998. The dynamics of EuroAtlantic blocking onsets. Q. J. R. Meteorol. Soc. 124, 1045-1070.

Mullen, S. L. 1986. The local balances of vorticity and heat for blocking anticyclones in a spectral general-circulation model. J. Atmos. Sci. 43, 1406-1441.

Nakamura, H. 1992. Midwinter suppression of baroclinic wave activity in the Pacific. J. Atmos. Sci. 49, 1629-1642.
Nakamura, H. 1994. Rotational evolution of potential vorticity associated with a strong blocking flow configuration over Europe. Geophys. Res. Lett. 21, 2003-2006.

Nakamura, H. and Fukamachi, T. 2004. Evolution and dynamics of summertime blocking over the Far East and the associated surface Okhotsk high. Q. J. R. Meteorol. Soc. 130, 1213-1233.

Nakamura, H. and Wallace, J. M. 1993. Synoptic behavior of baroclinic eddies during the blocking onset. Mon. Wea. Rev. 121, 1892-1903.

Nakamura, H., Nakamura, M. and Anderson, J. L. 1997. The role of high- and low-frequency dynamics in blocking formation. Mon. Wea. Rev. 125, 2074-2093.

Orlanski, I. 2003. Bifurcation in eddy life cycles: implications for storm track variability. J. Atmos. Sci. 60, 993-1023.

Pelly, J. L. and Hoskins, B. J. 2003. A new perspective on blocking. J. Atmos. Sci. 60, 743-755.

Polvani, L. and Esler, J. G. 2007. Transport and mixing of chemical airmasses in idealized baroclinic life cycles. J. Geophys. Res. 112, D23102, doi:10.1029/2007JD008555.

Schwierz, C. 2001. Interactions of Greenlands-Scale Orography and Extra-Tropical Synoptic-Scale Flow. Phd Dissertation ETH No. 14356. Swiss Federal Institute of Technology .

Schwierz, C., Croci-Maspoli, M. and Davies, H. C. 2004a. Perspicacious indicators of atmospheric blocking. Geophys. Res. Lett. 31, Art. No. L06125.

Schwierz, C., Dirren, S. and Davies, H. C. 2004b. Foreced waves on a zonally aligned jet stream. J. Atmos. Sci. 61, 73-87.

Shutts, G. J. 1983. The propagation of eddies in diffluent jetstreams eddy vorticity forcing of blocking flow-fields. Q. J. R. Meteorol. Soc. 109, 737-761.

Swanson, K. L. 2001. Blocking as a local instability to zonally varying flows. Q. J. R. Meteorol. Soc. 127, 1341-1355.

Swanson, K. L., Kushner, P. and Held, I. 1997. Dynamics of barotropic storm tracks. J. Atmos. Sci. 54, 791-810.

Thorncroft, C. D., Hoskins, B. J. and McIntyre, M. F. 1993. 2 paradigms of baroclinic-wave life-cycle behavior. Q. J. R. Meteorol. Soc. 119, $17-55$.

Tibaldi, S. and Molteni, F. 1990. On the operational predictability of blocking. Tellus 42A, 343-365.

Uppala, S. M., Kåallberg, P. W., Simmons, A. J., Andrae, U., Bechtold, V. D. and co-authors. 2005. The ERA-40 re-analysis. Q. J. R. Meteorol. Soc. 131, 2961-3012.

Vautard, R. and Legras, B. 1988. On the source of midlatitude lowfrequency variability, part 2: nonlinear equilibration of weather regimes. J. Atmos. Sci. 45, 2845-2867.

Wernli, H. and Sprenger, M. 2007. Identification and ERA15 Climatology of potential vorticity streamers and cut-offs near the extratropical tropopause. J. Atmos. Sci. 64, 1569-1586. 